\title{
A Hot-Hole Transport Model Based on Spherical Harmonics Expansion of the Anisotropic Bandstructure
}

\author{
H. KOSINA* and M. HARRER \\ Institute for Microelectronics, TU Vienna, Gusshausstrasse 27-29, A-1040 Vienna, Austria
}

\begin{abstract}
To represent the valence bands of cubic semiconductors a coordinate transformation is proposed such that the hole energy becomes an independent variable. This choice considerably simplifies the evaluation of the integrated scattering probability and the choice of the state after scattering in a Monte Carlo procedure. In the new coordinate system, a numerically given band structure is expanded into a series of spherical harmonics. This expansion technique is capable of resolving details of the band structure at the Brillouin zone boundary and hence can span an energy range of several electron-volts. Results of a Monte Carlo simulation employing the new band representation are shown.
\end{abstract}

Keywords: Monte Carlo Method, Hot Carrier, Transport, Semiconductor, Valence Band, Modeling

\section{INTRODUCTION}

Efforts on numerical modeling of hot carrier transport published to date deal mainly with hot electrons. One reason might be that for electrons some important transport properties are readily revealed by assuming simple effective-mass band models. For holes, however, an effective mass approximation is poor even very close to the $\Gamma$-point. Non-parabolicity is very pronounced and cannot be described by simple analytic expressions. The warped-band model [1], which is essentially parabolic, cannot be implemented in the Monte Carlo technique without additional simplifications [2].

The representation of the valence bands we present is specifically tailored to the needs of Monte Carlo transport calculations. These needs include efficient calculation of the scattering integrals and a straight- forward algorithm for the choice of the state after scattering.

\section{REPRESENTATION OF THE BANDSTRUCTURE}

To obtain the total scattering rate the transition probability given by Fermi's Golden rule has to be integrated in the three-dimensional k-space. Because of the energy-conserving $\delta$-function in the transition probability a coordinate transformation is desirable such that energy becomes one of the integration variables. Assume that the band structure is given in polar coordinates : $\varepsilon=\mathscr{E}(k, \Omega)$. We now introduce a coordinate transformation $(k, \Omega) \rightarrow(\varepsilon, \Omega)$ by inverting the function $\mathscr{E}(k, \Omega)$ with respect to $k$. The result of such an inversion is a function $\mathcal{K}$ describing equi-energy

\footnotetext{
* Corresponding author. Tel: +43 1 58801-3719. Fax:+431 5059224. E-mail: kosina@iue.tuwien.ac.at
} 
surfaces in k-space : $k=\mathcal{K}(\varepsilon, \Omega)$. Inversion of a function is possible only in an interval where the function is monotonous. By inspection of the full band structure one finds that both the heavy hole and split-off bands can entirely be represented by such functions $\mathcal{K}$ Above a hole energy of $E_{X}(3.04 \mathrm{eV})$ inversion of the light hole band is no longer unique.

In this work, we represent the function $\mathcal{X}$ as a series of spherical harmonics.

$$
\begin{array}{r}
K_{b}(\varepsilon, \Omega)^{3}=\frac{3}{4 \pi} \sum_{l=0}^{\infty} \sum_{m=0}^{l} a_{b, l m}(\varepsilon) P_{l}^{m}(\cos \theta) \cos m \phi, \\
b=\mathrm{H}, \mathrm{L}, \mathrm{SO}
\end{array}
$$

Derivation of the scattering rates is considerably eased by taking the third power of $\mathcal{K}$ as the function to be expanded. For symmetry reasons non-vanishing coefficients only exist for even values of $l$ and for $m$ being a multiple of 4 . With (1) a set of functions $a_{b, l m}(\varepsilon)$ contains the whole band structure information.

The density of states of a band represented by (1) is solely determined by the zero order coefficient.

$$
g_{b}(\varepsilon)=\frac{1}{4 \pi^{3}} \frac{d}{d \varepsilon} a_{b, 00}(\varepsilon), \quad b=\mathrm{H}, \mathrm{L}, \mathrm{SO}
$$

\section{SCATTERING RATES}

Wihthin this framework, we derived the scattering rates for acoustic deformation potential (ADP) scattering in the elastic approximation, optic deformation potential (ODP) scattering and ionized impurity scattering (ION) in the Brooks and Herring formalism.

$$
\begin{gathered}
\lambda_{i j}^{A D P}(\varepsilon)=\frac{D_{A}^{2} k_{B} T_{L}}{8 \pi^{2} \hbar \rho v_{s}^{2}} \frac{d}{d \varepsilon} a_{j, 00}(\varepsilon) \\
\lambda_{i j}^{O D P}(\varepsilon)=\frac{3 D_{o}^{2}}{2^{4} \pi^{2} \rho \omega_{o p}}\left(\begin{array}{c}
N_{o p} \\
N_{o p}+1
\end{array}\right) \frac{d}{d \varepsilon} a_{j, 00}\left(\varepsilon \pm h \omega_{0}\right) \\
\lambda_{i j}^{I O N}(\varepsilon, \Omega)=\frac{Z^{2} N_{l} \varepsilon^{4}}{4 \pi^{2} \hbar\left(\varepsilon_{0} \varepsilon_{r}\right)^{2}} \frac{1}{\left(2 k_{i}\left\langle k_{j}\right\rangle\right)^{2}} \\
\sum_{l=0}^{\infty} h_{l}^{i j}(\varepsilon) \sum_{m=0}^{l} \frac{d}{d \varepsilon} a_{j, l m}(\varepsilon) P_{l}^{m}(\cos \theta) \cos m \phi
\end{gathered}
$$

All these mechanisms induce both intraband and interband transitions. Other than for electrons, overlap integrals cannot be neglected for holes. The used approximations are of the form $G_{i i}=\frac{1}{4}\left(1+3 \cos ^{2} \beta\right)$ and $G_{i j}=\frac{3}{4} \sin ^{2} \beta$. The Coulomb scattering rate, which additionally depends on the solid angle of the wave vector, is expressed as a series of spherical harmonics. In Eq. (5), $\left\langle k_{j}\right\rangle$ denotes an average value over the solid angle, which is defined as $\left\langle k_{j}\right\rangle=\left(\frac{3}{4 \pi} a_{j, 00}(\varepsilon)\right)^{1 / 3}$. The coefficients $h_{l}^{i j}(\varepsilon)$ being a result of integration can be expressed in terms of Legendre functions of the second kind.

The distribution functions of the solid angle after scattering are given as spherical harmonics series. In a Monte Carlo procedure, the after scattering state can be chosen according to these distributions by a simple rejection technique.

\section{RESULTS AND DISCUSSION}

In this work, we use the series expansion (1) to represent the heavy and light hole bands up to $\varepsilon_{\text {hole }}=$ $3.04 \mathrm{eV}$, which is the band-energy at the $\mathrm{X}$-points. The numerical band structure has been computed by a nonlocal empirical pseudopotential method.

The functions $a_{h, l m}(\varepsilon)$ are represented numerically by means of a finite element method. To ensure continuous derivatives shape functions of third order have been chosen. The unknowns associated with the nodes of the energy grid have been determined by a variational approach [3]. From numerical band data the functions $a_{b, l m}(\varepsilon)$ can well be computed for nonvanishing hole energies, but not for an energy of zero. To obtain the $a_{b, l m}(0)$ we expand the expression for the warped band approximation. In this way, our band model combines the warped band approximation in the vicinity of the $\Gamma$-point where not enough numerical data points are available, and the numerical band structure for higher hole energies. 


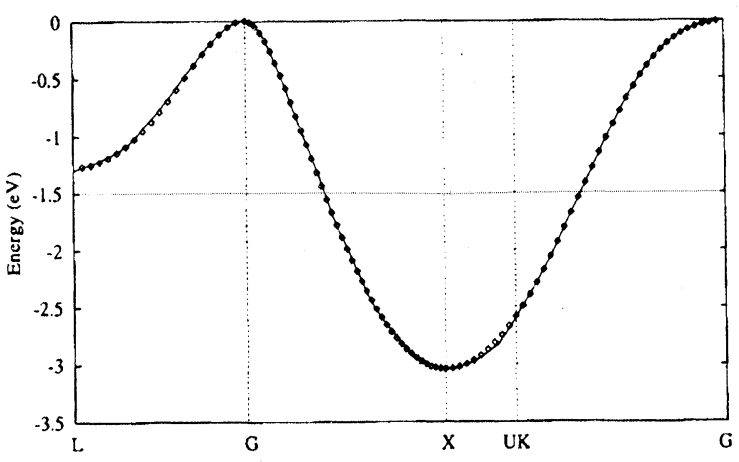

FIGURE 1 Comparison of numerical band structure (symbols) and the spherical harmonics expansion (lines) for the heavy hole band

Figure 1 shows the band diagram for the heavy hole band of silicon. Symbols refer to the data points of the numerical band structure, solid lines to the series expansion. Equi-energy lines in k-space are plotted in Figure 2. It turned out that at low energies less harmonics are required than at high energies. Therefore, we make the number of harmonics a function of energy. For instance, for the light hole band $l_{\max }=20$ at $0.5 \mathrm{eV}$, and $l_{\max }=60$ at $3.0 \mathrm{eV}$. The weak ripples at $3.0 \mathrm{eV}$ indicate that some higher order harmonics are still missing. In general, the higher the number of harmoncis, the better the details of the band structure can be resolved at the boundary of the Brillouin zone. On the other hand, for hole energies below $E_{L}(1.27 \mathrm{eV})$, where the band structure does not yet touch the zone boundary, a lower value of $l_{\max }$ is sufficient (typically $l_{\text {max }} \leq 28$ ).

As can be seen in Figure 2 the series representation provides states outside the first Brillouin zone which do not exist in reality. These artificial states yield an increased density of states and hence increased scattering rates. In the Monte Carlo procedure, scattering events to such artificial states outside the Brillouin zone are rejected and self-scattering is performed instead.

In Figure 3 the simulated drift velocity is compared to measured data [4]. The split-off band has been neglected in this simulation.

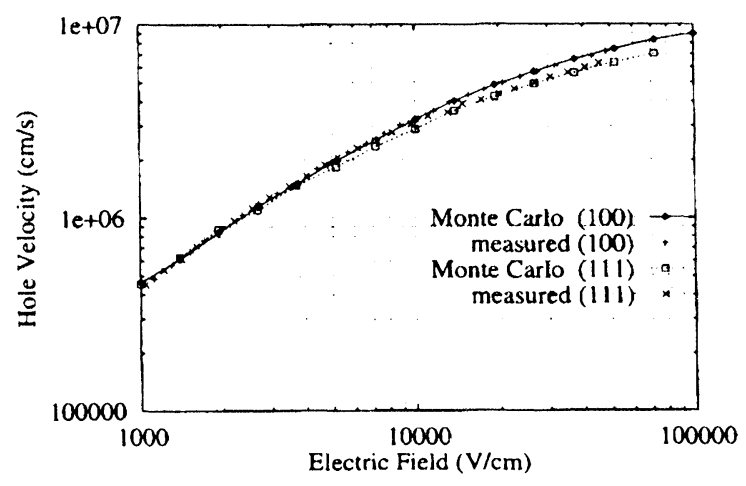

FIGURE 3 Comparison of simulated and measured [1] hole drift velocities as function of the electric field at $300 \mathrm{~K}$
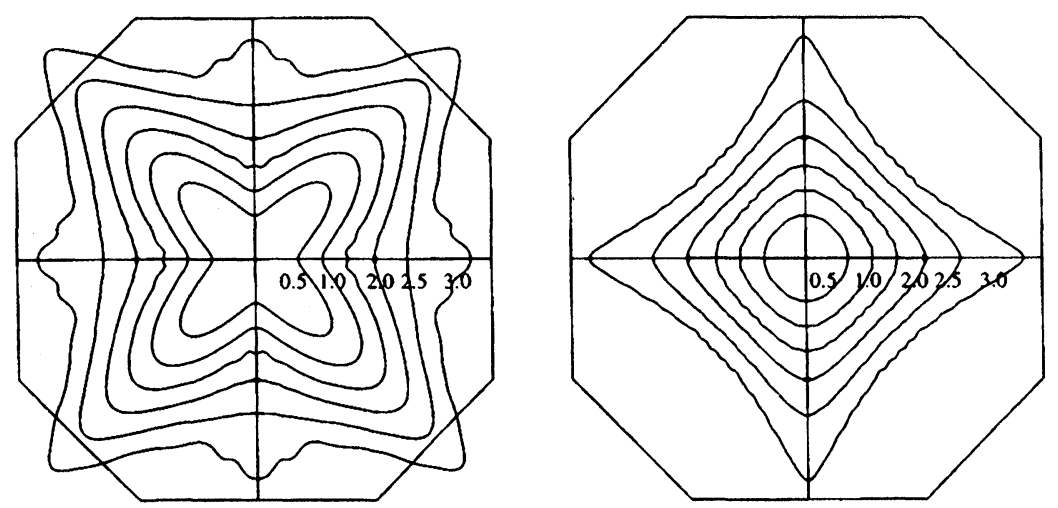

FIGURE 2 Cross section through the heavy hole (left) and light hole (right) bands from $0.5 \mathrm{eV}$ to $3.0 \mathrm{eV}$ in $0.5 \mathrm{eV}$ steps. The surrounding octagon indicates the boundary of the Brillouin zone 


\section{CONCLUSION}

A new method to represent numerical valence band data for Monte Carlo transport calculations has been developed. A function basically describing equienergy surfaces in k-space is expanded into a series of spherical harmonics. Depending on the energy range accounted for and the number of harmonics invoked the model can be considered either as an improved analytical band model or as a full-band model. In this work we demonstrated the full-band capabilities for hole energies up to $E_{X}(3.04 \mathrm{eV})$.

\section{References}

[1] K. Seeger, Semiconductor Physics. Springer, 1989.

[2] C. Jacoboni and L. Reggiani, "The Monte Carlo Method for the Solution of Charge Transport in Semiconductors with Applications to Covalent Materials," Rev.Mod.Phys., vol. 55, no. 3, pp. 645-705, 1983.

[3] W. Vetterling and S. Teukolsky, Numerical Recipes. Cambridge University Press, 1986.

[4] C. Canali, G. Ottaviani, and A. Quaranta, "Drift Velocity of Electrons and Holes and Associated Anisotropic Effects in Silicon," J.Phys.Chem.Solids, vol. 32, no. 8, pp. 1707-1720, 1971. 

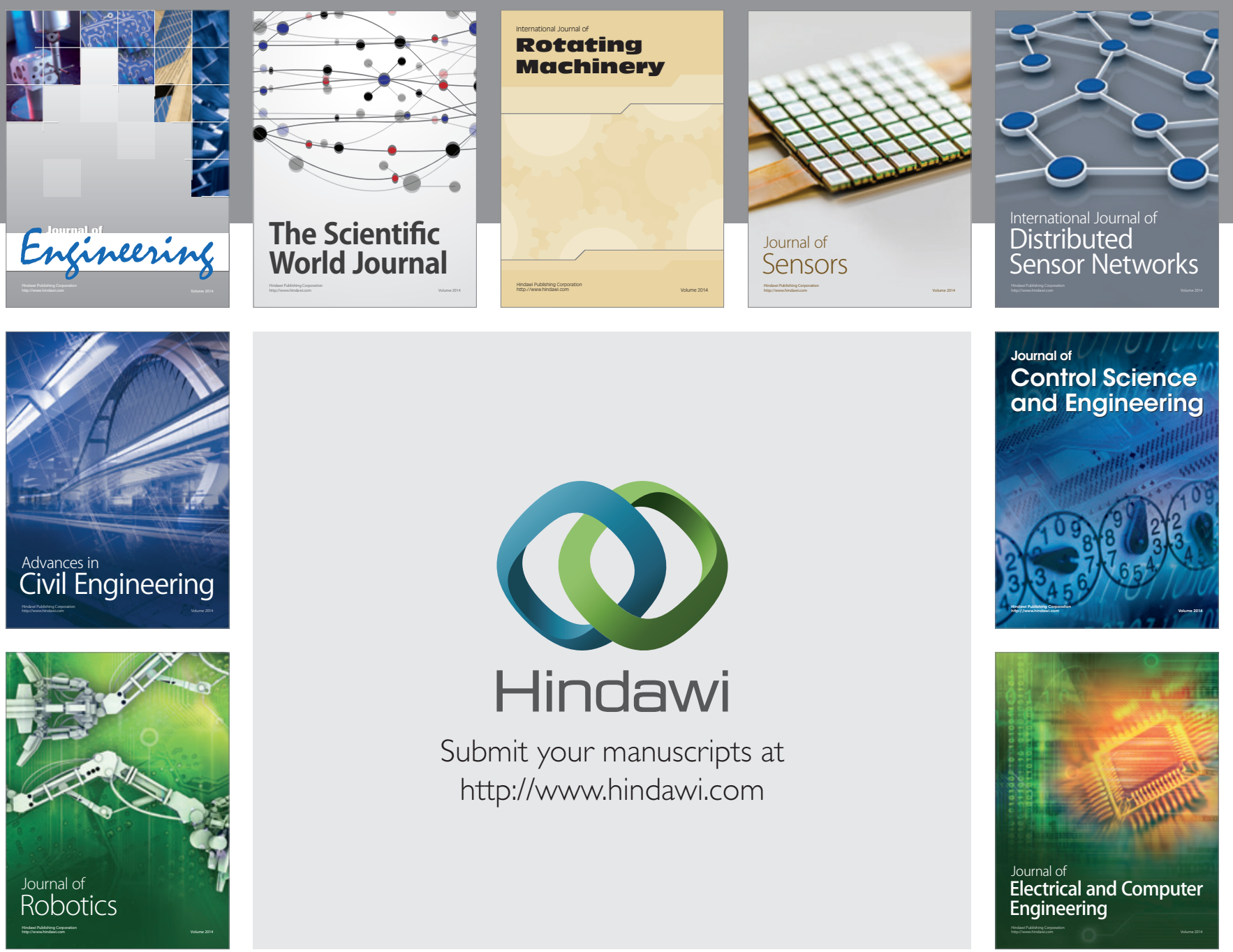

Submit your manuscripts at

http://www.hindawi.com
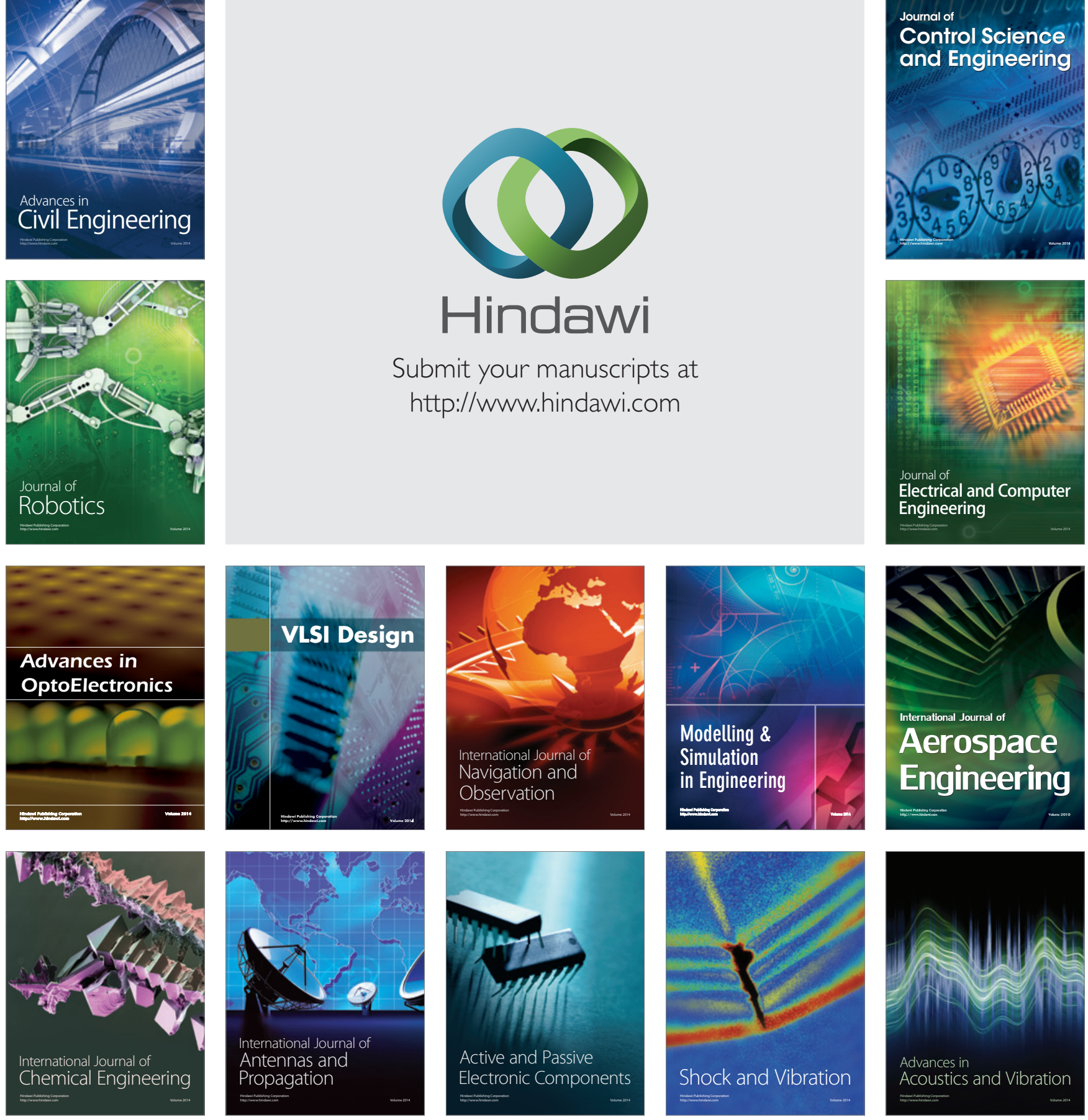